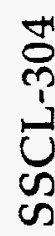

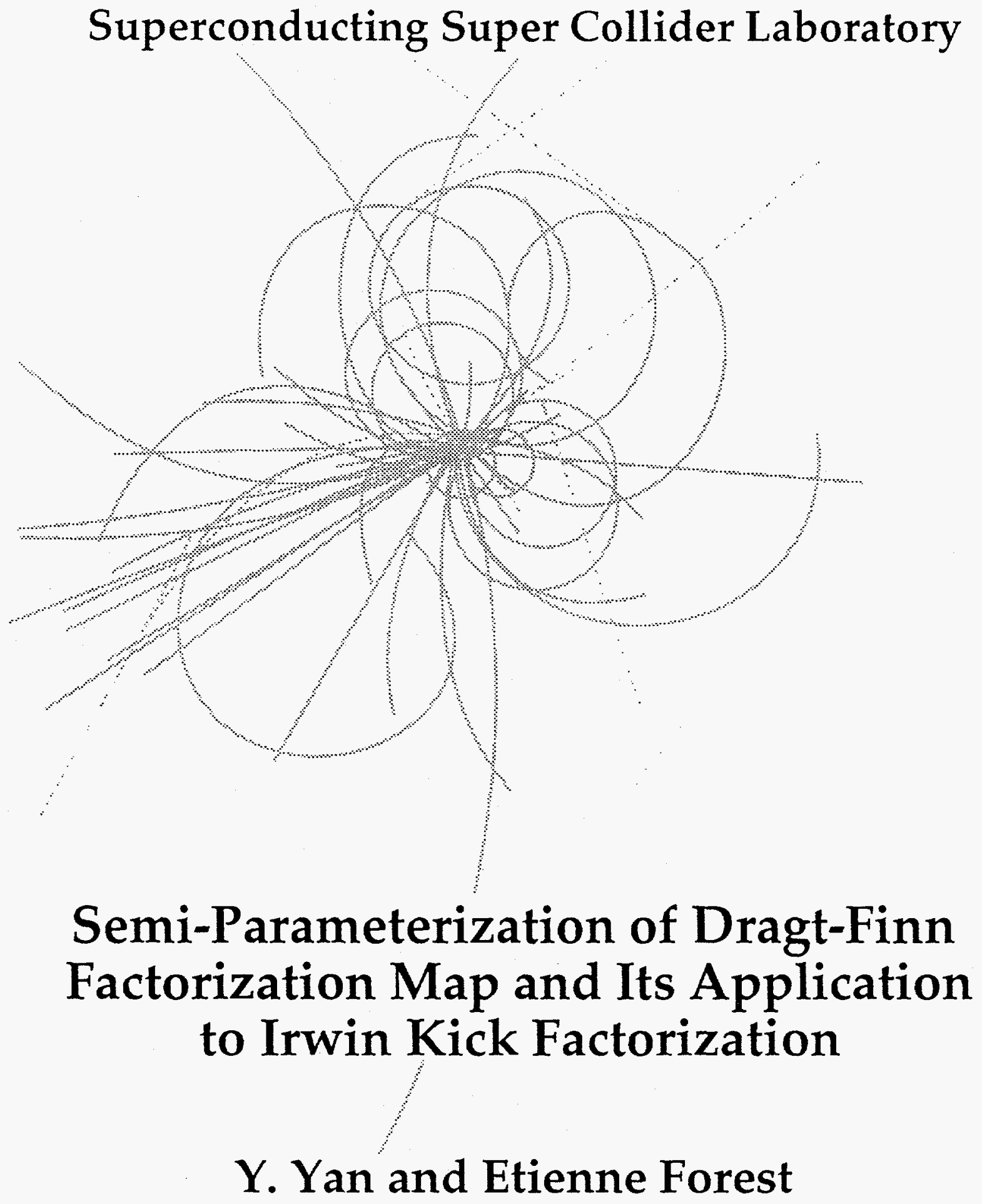

November 1990

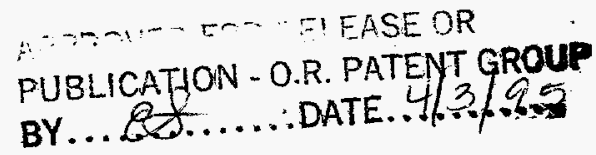




\section{DISCLAIMER}

Portions of this document may be illegible in electronic image products. Images are produced from the best available original document. 


\title{
SEMI-PARAMETERIZATION OF DRAGT-FINN FACTORIZATION MAP AND ITS APPLICATION TO IRWIN KICK FACTORIZATION
}

\author{
Yiton Yan \\ Superconducting Super Collider Laboratory* \\ Accelerator Design and Operating Divsion \\ 2550 Beckleymeade Avenue \\ Dallas, TX 75237 \\ and \\ Etienne Forest \\ Lawrence Berkeley Laboratory \\ 1 Cyclotron Road \\ Berkeley, CA 94720
}

November, 1990

\footnotetext{
" Operated by Universities Research Association, Inc., for the U.S. Department of Energy under Contract No. DE-AC02-89ER40486.
} 
SSCL-304

\title{
Semi-Parameterization of Dragt-Finn Factorization Map and its Application to Irwin Kick Factorization
}

\author{
Yiton Yan \\ Superconducting Super Collider Laboratory* \\ Accelerator Design and Operating Divsion \\ 2550 Beckleymeade Avenue \\ Dallas, TX 75237 \\ and \\ Etienne Forest \\ Lawrence Berkeley Laboratory \\ 1 Cyclotron Road \\ Berkeley, CA 94720 \\ November, 1990
}

\begin{abstract}
This note is based on the work we did in August, 1989 for parameterizing Irwin factorization kick map. A semi-parameterization method was employed in which the linear part of the map is three-dimensional (six-dimensional in phase space) while the nonlinear part of the map is two-and-one-half-dimensional. The one-half dimension can be eliminated once the parameter (off-momentum) is updated at the beginning of each turn of the kick map.
\end{abstract}

* Operated by Universities Research Association, Inc., for the U.S. Department of Energy under Contract No. DE-AC02-89ER40486. 


\section{INTRODUCTION}

In 1989, Irwin proposed a method of converting a Dragt-Finn factorization [1] of a Taylor map into kicks. [2] This method is different from another method [3] in that only the minimized highest-order rotation bases are used as the bases for all orders while Lagrange multipliers are employed for (a) eliminating the extra independent bases in order to obtain a unique solution, and (b) suppressing the higher-order (order higher than the order of the original Taylor map) artificial terms. If the artificial terms can be suppressed enough and be negligible, then this method may be promising for fast long-term tracking. Therefore, an investigation was started right after Irwin's proposal to verify its applicability for the SSC long-term tracking. In order to enhance tracking speed, a semi-parameterization method was employed to parameterize the nonlinear part of the Dragt-Finn factorization map and therefore reducing from three dimensions to two dimensions for the kicks proposed by Irwin. To accomplish this, the one-turn Taylor map should not involve any RF cavity so that the off-momentum (represented by the energy deviation $\delta=\Delta E / E$ ) be kept invariant and so be treated as a parameter. To update the off-momentum, one simply track the particles over the RF cavities separately.

Let a closed-orbit Taylor map (can be extracted using Zmap [4] for the SSC) be extracted up to the $\Omega$ order for a beam line from after an RF cavity to before the next RF cavity. The map can be expressed as follows (we adapt the same notational convertion as in ZLIB [5] manual):

$$
m: \overrightarrow{\mathbf{z}}=\overrightarrow{\mathbf{U}}(\overrightarrow{\mathbf{z}})=M \overrightarrow{\mathbf{z}}+\sum_{k=2}^{\Omega} \overrightarrow{\mathbf{U}}^{k}(\overrightarrow{\mathbf{z}}-)
$$

where the transpose of the coordinates are

$$
\begin{aligned}
\overrightarrow{\mathbf{z}}^{T} & =\left[z_{1}, z_{2}, \ldots, z_{n}\right]=\left[\overrightarrow{\mathbf{x}}^{T}, \delta, P_{\delta}\right], \\
\overrightarrow{\mathbf{x}}^{T} & =\left[x, P_{x}, y, P_{y}\right],
\end{aligned}
$$


and the transpose of the VTPS (vector truncated power series) is

$$
\overrightarrow{\mathbf{U}}^{T}(\overrightarrow{\mathbf{z}})=\left[U_{1}(\overrightarrow{\mathbf{z}}), U_{2}(\overrightarrow{\mathbf{z}}), U_{3}(\overrightarrow{\mathbf{z}}), U_{4}(\overrightarrow{\mathbf{z}}), U_{5}(\overrightarrow{\mathbf{z}}), U_{6}(\overrightarrow{\mathbf{z}})\right]
$$

Note that $P_{x}, P_{y}$, and $P_{\delta}$ are the conjugate momenta of $x, y$, and $\delta$ respectively. $U_{5}(\overrightarrow{\mathbf{z}})=\delta$, that is, the off-momentum, $\delta$, is an invariant since no RF cavity is involved for acceleration. The nonlinear part of the map are only dependent on $\overrightarrow{\mathbf{x}}\left(\overrightarrow{\mathbf{x}} \equiv\left[x, P_{x}, y, P_{y}\right]\right)$ and $\delta$, not on $P_{\delta}$ as we have explicitly expressed $\overrightarrow{\mathbf{U}}^{k}(\overrightarrow{\mathbf{z}}-)$ for $k>1$ as nonlinear VTPS of $\overrightarrow{\mathbf{z}}-\equiv\left[x, P_{x}, y, P_{y}, \delta\right]$. Furthermore, each of the constant term in $U_{i}(i=1,2, \ldots, 6)$ is 0 .

\section{BLOCK DIAGONALIZATION OF $M$}

Before the nonlinear Dragt-Finn factorization can be performed, block diagonalization and similarity transformation are necessary such that the transformed map is semi-normalized in the linear part. In order to parameterize the nonlinear Dragt-Finn generators, the transformed nonlinear part of the map should be independent of $P_{\delta}$ and the transformed $U_{5}^{k}(\overrightarrow{\mathbf{z}})$ should be 0 for $k=2, \Omega$. We describe, in this section, the method we used to accomplish this.

The linear part of the Taylor map can be generally expressed as a symplectic matrix given by

$$
M=\left(\begin{array}{ccc}
N_{4 \times 4} & \overrightarrow{\mathbf{m}} & \overrightarrow{0} \\
\overrightarrow{\mathbf{0}}^{T} & 1 & 0 \\
\overrightarrow{\mathbf{n}}^{T} & \alpha & 1
\end{array}\right)
$$

with the constraint that $M$ and $N(N$ is a $4 \times 4$ matrix) are both symplectic, that is, $M^{T} S M=S=S_{6 \times 6}$ and $N^{T} S N=S=S_{4 \times 4}$, where $\overrightarrow{\mathbf{m}}$ and $\overrightarrow{\mathbf{n}}$ are vectors with 4 elements and

$$
S_{6 \times 6}=\left(\begin{array}{ccc}
S_{2 \times 2} & O_{2 \times 2} & O_{2 \times 2} \\
O_{2 \times 2} & S_{2 \times 2} & O_{2 \times 2} \\
O_{2 \times 2} & O_{2 \times 2} & S_{2 \times 2}
\end{array}\right), \quad S_{4 \times 4}=\left(\begin{array}{cc}
S_{2 \times 2} & O_{2 \times 2} \\
O_{2 \times 2} & S_{2 \times 2}
\end{array}\right)
$$


with

$$
S_{2 \times 2}=\left(\begin{array}{cc}
0 & -1 \\
1 & 0
\end{array}\right), \quad \text { and } \quad O_{2 \times 2}=\left(\begin{array}{ll}
0 & 0 \\
0 & 0
\end{array}\right)
$$

First, we found the first-order parameter- $(\delta-)$ dependent closed orbit $\overrightarrow{\mathbf{x}}_{c}$ by imposing that

$$
\overrightarrow{\mathbf{x}}_{c}=N \overrightarrow{\mathbf{x}}_{c}+\delta \overrightarrow{\mathbf{m}}
$$

and so

$$
\overrightarrow{\mathbf{x}}_{c}=(I-N)^{-1} \delta \overrightarrow{\mathbf{m}}=\vec{\xi} \delta
$$

where

$$
\vec{\xi}=(I-N)^{-1} \overrightarrow{\mathbf{m}}
$$

Then, we found a symplectic generation matrix $M_{1}$ to transform the coordinates $\overrightarrow{\mathbf{x}}$ into the linear-order- $\delta$-dependent-closed-orbit coordinates while keeping the parameter, $\delta$, invariant. $P_{\delta}$ is inevitably transformed to have $M_{1}$ symplectic, i.e. to have $M_{1}^{T} S M_{1}=S$. The generator $M_{1}$ is given by

$$
M_{1}=\left(\begin{array}{ccc}
I_{4 \times 4} & \vec{\xi} & \overrightarrow{0} \\
\overrightarrow{0}^{T} & 1 & 0 \\
\vec{\xi}^{T} S & 0 & 1
\end{array}\right)
$$

and its inverse is given by

$$
M_{1}^{-1}=\left(\begin{array}{ccc}
I_{4 \times 4} & -\vec{\xi} & \overrightarrow{0} \\
\overrightarrow{0}^{T} & 1 & 0 \\
-\vec{\xi}^{T} S & 0 & 1
\end{array}\right)
$$

where $S=S_{4 \times 4}$. The forms of the generator $M_{1}$ and its inverse $M_{1}^{-1}$ show that through the similarity transformation, we would still have the two required properties ( $\delta$ is an invariant and $\overrightarrow{\mathrm{x}}$ is independent of $P_{\delta}$ ) for parameterizing the 
nonlinear Dragt-Finn generators. Performing the similarity transformation we have

$$
m^{\prime}: \overrightarrow{\mathbf{z}}=\left(\mathcal{M}_{1} m \mathcal{M}_{1}^{-1}\right): \overrightarrow{\mathbf{z}}=\left(M_{1}^{-1} M M_{1}\right) \overrightarrow{\mathbf{z}}+\sum_{k=2}^{\Omega} M_{1}^{-1} \overrightarrow{\mathrm{U}}^{k}\left(M_{1} \overrightarrow{\mathbf{z}}\right)
$$

or simply,

$$
m^{\prime}: \overrightarrow{\mathbf{z}}=M^{\prime} \overrightarrow{\mathbf{z}}+\sum_{k=2}^{\Omega} \overrightarrow{\mathbf{U}}^{\prime k}(\overrightarrow{\mathbf{z}})
$$

where

$$
M^{\prime}=M_{1}^{-1} M M_{1}=\left(\begin{array}{ccc}
N_{4 \times 4} & \overrightarrow{0} & \overrightarrow{0} \\
\overrightarrow{0}^{T} & 1 & 0 \\
\overrightarrow{0}^{T} & \alpha_{c} & 1
\end{array}\right)
$$

and $\alpha_{c}=\alpha+\overrightarrow{\mathbf{n}}^{T} S \vec{\xi}$ is the momentum compaction. Note that $\mathcal{M}_{1}$ and $\mathcal{M}_{1}^{-1}$ are the symplectic transformation generator representing the matrices $M_{1}$ and $M_{1}^{-1}$ respectively. However, for convenience, we have followed the convention that $\mathcal{M}_{1}$ and $\mathcal{M}_{1}^{-1}$ operate on the global variables while their matrix representations, $M_{1}$ and $M_{1}^{-1}$ operate on local variables. Please also note that $U_{5}^{\prime k}(\overrightarrow{\mathbf{z}})=0$ and $\overrightarrow{\mathbf{U}}^{\prime k}(\overrightarrow{\mathbf{z}})$ is independent of $P_{\delta}$ for $k \geq 2$.

Now that the transverse linear map $N$ is decoupled from the longitudinal space, the linear part of the map, $M^{\prime}$, can be semi-normalized by another similarity transformation as follows: 


$$
\begin{aligned}
\left(\begin{array}{ccc}
R_{4 \times 4} & \overrightarrow{0} & \overrightarrow{0} \\
\overrightarrow{0}^{T} & 1 & 0 \\
\overrightarrow{0}^{T} & \alpha_{c} & 1
\end{array}\right) & =M_{R}=M_{1}^{\prime-1} M^{\prime} M_{1}^{\prime} \\
& =\left(\begin{array}{ccc}
N_{1}^{-1} & \overrightarrow{0} & \overrightarrow{0} \\
\overrightarrow{0}^{T} & 1 & 0 \\
\overrightarrow{0}^{T} & 0 & 1
\end{array}\right)\left(\begin{array}{ccc}
N_{4 \times 4} & \overrightarrow{0} & \overrightarrow{0} \\
\overrightarrow{0}^{T} & 1 & 0 \\
\overrightarrow{0}^{T} & \alpha_{c} & 1
\end{array}\right)\left(\begin{array}{ccc}
N_{1} & \overrightarrow{0} & \overrightarrow{0} \\
\overrightarrow{0}^{T} & 1 & 0 \\
\overrightarrow{0}^{T} & 0 & 1
\end{array}\right) \\
& =\left(\begin{array}{ccc}
N_{1}^{-1} N_{4 \times 4} N_{1} & \overrightarrow{0} & \overrightarrow{0} \\
\overrightarrow{0}^{T} & 1 & 0 \\
\overrightarrow{0}^{T} & \alpha_{c} & 1
\end{array}\right),
\end{aligned}
$$

where

$$
R_{4 \times 4}=N_{1}^{-1} N_{4 \times 4} N_{1}
$$

is the usual normalization for a 4 -dimensional symplectic matrix. The map is thus transformed as follows:

$$
{ }_{1} m: \overrightarrow{\mathbf{z}}=\left(\mathcal{M}_{1}^{\prime} m^{\prime} \mathcal{M}_{1}^{\prime-1}\right): \overrightarrow{\mathbf{z}}=\left(M_{1}^{\prime-1} M^{\prime} M_{1}^{\prime}\right) \overrightarrow{\mathbf{z}}+\sum_{k=2}^{\Omega} M_{1}^{\prime-1} \overrightarrow{\mathbf{U}}^{k}\left(M_{1}^{\prime} \overrightarrow{\mathbf{z}}\right),
$$

or simply,

$$
{ }_{1} m: \overrightarrow{\mathbf{z}}=M_{R} \overrightarrow{\mathbf{z}}+\sum_{k=2}^{\Omega}{ }_{1} \overrightarrow{\mathbf{U}}^{k}(\overrightarrow{\mathbf{z}}-)
$$

The form of the matrix $M_{R}$, which has already been decoupled between the transverse coordinates and the longitudinal coordinates, guarantees that an additional transformation by operating $M_{R}^{-1}$ on the map would not destroy the two requirements ( $\delta$ is an invariant and non-linear part of the map is independent of $P_{\delta}$ ) for parameterizing the nonlinear Lie generators. So we made another 
transformation by operating $\mathcal{M}_{R}^{-1}$ on the map and obtained

$$
{ }_{2} m: \overrightarrow{\mathbf{z}}=\left(\mathcal{M}_{R}^{-1}{ }_{1} m\right): \overrightarrow{\mathbf{z}}=\overrightarrow{\mathbf{z}}+\sum_{k=2}^{\Omega} \overrightarrow{\mathrm{U}}^{k}\left(M_{R}^{-1} \overrightarrow{\mathbf{z}}\right)
$$

or simply,

$$
{ }_{2} m: \overrightarrow{\mathbf{z}}=\overrightarrow{\mathbf{z}}+\sum_{k=2}^{\Omega} \overrightarrow{\mathrm{U}}^{k}(\overrightarrow{\mathbf{z}}-)
$$

Note that $\mathcal{M}_{R}^{-1}$ operate on the global variables and its matrix representation $M_{R}^{-1}$ operate on the local variables. ${ }_{2} U_{5}^{k}(\overrightarrow{\mathbf{z}})=0$ and ${ }_{2} \vec{U}^{k}(\overrightarrow{\mathbf{z}}-)$ is independent of $P_{\delta}$ for $k \geq 2$.

\section{DRAGT-FINN FACTORIZATION}

We reformed Eq. (1) as follows

$$
{ }_{2} m: \overrightarrow{\mathbf{z}}=\overrightarrow{\mathbf{z}}+{ }_{2} \overrightarrow{\mathbf{U}}^{2}(\overrightarrow{\mathbf{z}}-)+\sum_{k=3}^{\Omega} \overrightarrow{\mathbf{U}}^{k}(\overrightarrow{\mathbf{z}}-)
$$

where $\overrightarrow{\mathbf{z}}-=[\overrightarrow{\mathbf{x}}, \delta]$ indicates that ${ }_{2} \overrightarrow{\mathrm{U}}^{k}(\overrightarrow{\mathbf{z}}-)$ is independent of $P_{\delta}$.

To obtain the second-order Lie generator, since ${ }_{2} U_{5}^{2}(\overrightarrow{\mathbf{z}}-)=0$, we let

$$
\left[f_{2}(\overrightarrow{\mathbf{z}}-), \overrightarrow{\mathbf{z}}\right]={ }_{2} \overrightarrow{\mathrm{U}}^{2}(\overrightarrow{\mathbf{z}}-),
$$

so

$$
\partial f_{2}(\overrightarrow{\mathbf{z}}-) / \partial \overrightarrow{\mathbf{z}}=-S_{2} \overrightarrow{\mathbf{U}}^{2}(\overrightarrow{\mathbf{z}}-)
$$

and so

$$
f_{2}(\overrightarrow{\mathbf{z}}-)=-\overrightarrow{\mathbf{z}}^{T} S_{2} \overrightarrow{\mathbf{U}}^{2}(\overrightarrow{\mathbf{z}}-) / 3
$$

where

$$
S=S_{6 \times 6}
$$


Note that the second-order Lie generator, $f_{2}(\overrightarrow{\mathbf{z}}-)$, is a third-order polynomial of $\overrightarrow{\mathbf{z}}$-. Note that $f_{2}(\overrightarrow{\mathbf{z}}-)$ is independent of $P_{\delta}$. Since

$$
\exp \left(: f_{2}(\overrightarrow{\mathbf{z}}-):\right) \overrightarrow{\mathbf{z}}=\overrightarrow{\mathbf{z}}+\left[f_{2}(\overrightarrow{\mathbf{z}}-), \overrightarrow{\mathbf{z}}\right]+\left[f_{2}(\overrightarrow{\mathbf{z}}-),\left[f_{2}(\overrightarrow{\mathbf{z}}), \overrightarrow{\mathbf{z}}\right]\right] / 2 !+\ldots,
$$

we let

$$
{ }_{2} m: \overrightarrow{\mathbf{z}}=\exp \left(: f_{2}(\overrightarrow{\mathbf{z}}-):\right) \overrightarrow{\mathbf{z}}+\sum_{k=3}^{\Omega} \Delta_{2} \overrightarrow{\mathbf{U}}^{k}(\overrightarrow{\mathbf{z}}-)
$$

where

$$
\sum_{k=3}^{\Omega} \Delta_{2} \overrightarrow{\mathbf{U}}^{k}(\overrightarrow{\mathbf{z}}-)={ }_{2} m: \overrightarrow{\mathbf{z}}-\exp \left(: f_{2}(\overrightarrow{\mathbf{z}}-):\right) \overrightarrow{\mathbf{z}}
$$

Then we obtained

$$
{ }_{3} m: \overrightarrow{\mathbf{z}}=\left\{\exp \left(-: f_{2}(\overrightarrow{\mathbf{z}}-):\right){ }_{2} m\right\}: \overrightarrow{\mathbf{z}}=\overrightarrow{\mathbf{z}}+{ }_{3} \overrightarrow{\mathbf{U}}^{3}(\overrightarrow{\mathbf{z}}-)+\sum_{k=4}^{\Omega}{ }_{3} \overrightarrow{\mathbf{U}}^{k}(\overrightarrow{\mathbf{z}}-)
$$

Eq. (4) is similar to Eq. (2). So we let $\left[f_{3}(\overrightarrow{\mathbf{z}}-), \overrightarrow{\mathbf{z}}\right]={ }_{3} \overrightarrow{\mathbf{U}}^{3}(\overrightarrow{\mathbf{z}}-)$ to obtain the third-order Lie generator given by $f_{3}(\overrightarrow{\mathbf{z}}-)=-\overrightarrow{\mathbf{z}}^{T} S_{3} \overrightarrow{\mathbf{U}}^{3}(\overrightarrow{\mathbf{z}}-) / 4$ and follow the similar process as we obtained Eq. (4), we obtained

$$
{ }_{4} m: \overrightarrow{\mathbf{z}}=\left\{\exp \left(-: f_{3}(\overrightarrow{\mathbf{z}}-):\right){ }_{3} m\right\}: \overrightarrow{\mathbf{z}}=\overrightarrow{\mathbf{z}}+{ }_{4} \overrightarrow{\mathbf{U}}^{4}(\overrightarrow{\mathbf{z}}-)+\sum_{k=5}^{\Omega}{ }_{4} \overrightarrow{\mathrm{U}}^{k}(\overrightarrow{\mathbf{z}}-),
$$

Through iteration, we finally obtained all the Lie generators

$$
f_{i}(\overrightarrow{\mathbf{z}}-)=-\overrightarrow{\mathbf{z}}^{T} S_{i} \overrightarrow{\mathbf{U}}^{i}(\overrightarrow{\mathbf{z}}-) /(i+1),
$$

and

$$
{ }_{i+1} m: \overrightarrow{\mathbf{z}}=\left\{\exp \left(-: f_{i}(\overrightarrow{\mathbf{z}}-):\right)_{i} m\right\}: \overrightarrow{\mathbf{z}}=\overrightarrow{\mathbf{z}}+{ }_{i+1} \overrightarrow{\mathbf{U}}^{i+1}(\overrightarrow{\mathbf{z}}-)+\sum_{k=i+2}^{\Omega}{ }_{i+1} \overrightarrow{\mathbf{U}}^{k}(\overrightarrow{\mathbf{z}}-),
$$


and so

$$
\begin{aligned}
& \Omega+1=: \overrightarrow{\mathbf{z}}=\left\{\exp \left(-: f_{\Omega}(\overrightarrow{\mathbf{z}}-):\right)_{\Omega} m\right\}: \overrightarrow{\mathbf{z}}=\overrightarrow{\mathbf{z}}+{ }_{\Omega+1} \overrightarrow{\mathbf{U}}^{\Omega+1}(\overrightarrow{\mathbf{z}}-)+\sum_{k=\Omega+2}^{\Omega} \overrightarrow{\mathbf{U}}^{k}(\overrightarrow{\mathbf{z}}-) \\
& =\overrightarrow{\mathbf{z}}+\sigma(\Omega+1) \approx \overrightarrow{\mathbf{z}}=I \overrightarrow{\mathbf{z}} .
\end{aligned}
$$

Therefore, neglecting terms with order higher than $\Omega$, we had (in terms of global variables)

$$
m=\mathcal{M}_{1}^{-1} \mathcal{M}_{1}^{\prime-1} \mathcal{M}_{R 2} m \mathcal{M}_{1}^{\prime} \mathcal{M}_{1} \approx \mathcal{M}_{1}^{-1} \mathcal{M}_{1}^{\prime-1} \mathcal{M}_{R} m_{f} \mathcal{M}_{1}^{\prime} \mathcal{M}_{1}
$$

where

$$
m_{f}=\exp \left(: f_{2}(\overrightarrow{\mathbf{z}}-):\right) \exp \left(: f_{3}(\overrightarrow{\mathbf{z}}-):\right) \ldots \exp \left(: f_{\Omega}(\overrightarrow{\mathbf{z}}-):\right)
$$

\section{KICK FACTORIZATION BASES}

Followed Irwin's Work, we first decided the total number of kicks necessary. Recall that each of the Dragt-Finn factors, $f_{i}(\overrightarrow{\mathbf{z}}-)$ 's, is independent of $P_{\delta}$, i.e. its longitudinal dimension $\left(\delta, P_{\delta}\right)$ has already been in a kick form, we only need rotation basees in the transverse dimension. Since the same rotation bases are used for each of the Dragt-Finn factors, $f_{i}(\overrightarrow{\mathbf{z}}-)$ 's, the minimum required number of kicks is determined by the highest-order of Dragt-Finn factor, $f_{\Omega}(\overrightarrow{\mathbf{z}}-)$, which is given by [2]

$$
K \geq \begin{cases}(\Omega+3)^{2} / 4 & \text { if } \Omega \text { is odd } \\ (\Omega+2)(\Omega+4) / 4 & \text { if } \Omega \text { is even }\end{cases}
$$

Once the total number, $K$, of rotation bases is chosen, the next step is to choose the rotation bases. We used random number generator to generate the two sets of rotation angles, $\theta_{x k}$ and $\theta_{y k}, k=1,2, \ldots, K$. Note that $0 \leq \theta_{x k}<\pi$ and $0 \leq \theta_{y k}<\pi$ for all $k$ 's. If two of the angles are too close in a set, that 
set of angles were regenerated until no two angles are too close based on a predetermined criterion. We then calculated the rotation bases, which were given by

$$
\begin{aligned}
& C_{x k}=\cos \theta_{x k}, \\
& S_{x k}=\sin \theta_{x k}, \\
& C_{y k}=\cos \theta_{y k}, \\
& S_{y k}=\sin \theta_{y k} .
\end{aligned}
$$

New local base coordinates were then formed by letting

$$
\begin{aligned}
& x_{k}=C_{x k} x+S_{x k} P_{x} \\
& y_{k}=C_{y k} y+S_{y k} P_{y}
\end{aligned}
$$

The task of this section is to construct from the Dragt-Finn factorization given in Eq. (8) a kick factorization of the form

$$
m_{g}=\exp \left(: g_{1}\left(\overrightarrow{\mathbf{x}}_{1}+\right):\right) \exp \left(: g_{2}\left(\overrightarrow{\mathbf{x}}_{2}+\right):\right) \ldots \exp \left(: g_{k}\left(\overrightarrow{\mathbf{x}}_{k}+\right):\right) \ldots \exp \left(: g_{K}\left(\overrightarrow{\mathbf{x}}_{K}+\right):\right)
$$

such that

$$
m_{g}=m_{f}+\sigma(\Omega+1) \approx m_{f}
$$

where $m_{f}$ is given in Eq. (8) and $\overrightarrow{\mathbf{x}}_{k}+$ is defined as $\overrightarrow{\mathbf{x}}_{k}+=\left(x_{k}, y_{k}, \delta\right)$ with the ' + ' to emphasize that the third dimension, $\delta$, although appears, has no effects in determining the rotation bases.

\section{KICK FACTORIZATION OF THE SECOND-ORDER DRAGT-FINN FACTOR}

The task is to find ${ }_{2} g_{k}\left(\vec{x}_{k}+\right)$, for $k=1,2, \ldots, K$ such that

$$
\sum_{k=1}^{K}{ }_{2} g_{k}\left(\overrightarrow{\mathbf{x}}_{k}+\right)=f_{2}(\overrightarrow{\mathbf{z}}-)
$$


that is,

$$
\sum_{k=1}^{K} \sum_{n+m+o=3} \beta_{k}^{n m o} x_{k}^{n} y_{k}^{m} \delta^{o}=\sum_{n+m+o=3} \sum_{r=0}^{n} \sum_{s=0}^{m} \alpha_{r s}^{n m o} x^{r} P_{x}^{n-r} y^{s} P_{y}^{m-s} \delta^{o}
$$

if we take the explicit power series for ${ }_{2} g_{k}\left(\overrightarrow{\mathbf{x}}_{k}+\right)$ and $f_{2}(\overrightarrow{\mathbf{z}}-)$. Therefore, the purpose is to find all the $\beta_{k}^{n m o}$ for $k=1,2, \ldots, K$, and for all possible combinations of $n, m, o$ such that $n+m+o=3$ (note that both of ${ }_{2} g_{k}\left(\overrightarrow{\mathbf{x}}_{k}+\right.$ ) and $f_{2}(\overrightarrow{\mathbf{z}}-)$ are 3rd-order polynomials). Simply speaking, for each possible combination of $n, m, o$, the task is to find all the $\beta_{k}^{n m o}$ for $k=1,2, \ldots, K$ such that

$$
\sum_{k=1}^{K} \beta_{k}^{n m o} x_{k}^{n} y_{k}^{m}=\sum_{r=0}^{n} \sum_{s=0}^{m} \alpha_{r s}^{n m o} x^{r} P_{x}^{n-r} y^{s} P_{y}^{m-s}
$$

Note that the third dimension, $\delta$, is a common factor on both sides of the "=" sign, and so has been taken out of the equation. Therefore we have exactly the same equation as Irwin's except an extra superscript, "o", appears for $\beta_{k}^{n m o}$ and $\alpha_{r s}^{n m o}$ to indicate that there are more coefficients than a non-parameterized case.

To solve Eq. (10) for $\beta_{k}^{n m o}, k=1,2, \ldots, K(n, m, o$ fixed), the key is to take $x_{k}^{n} y_{k}^{m}, k=1,2, \ldots(n+1)(m+1)$ as complete bases (can always be proved afterwards). Note that $K$ was chosen such that $(n+1)(m+1) \leq K$ for all possible combinations of $n, m, o$, such that $n+m+o \leq \Omega+1$. With the new complete bases, we can obtain

$$
x^{r} P_{x}^{n-r} y^{s} P_{y}^{m-s}=\sum_{k=1}^{(n+1)(m+1)} C_{r s k}^{n m o} x_{k}^{n} y_{k}^{m}
$$

for each combination of $r, s$, where $r=0,1, \ldots n$, and $s=0,1, \ldots m$, and

$$
x_{j}^{n} y_{j}^{m}=\sum_{k=1}^{(n+1)(m+1)} q_{j k}^{n m o} x_{k}^{n} y_{k}^{m}
$$


for each of the extra rotation bases $x_{j}^{n} y_{j}^{m}, j=(n+1)(m+1)+1, \ldots K$. Substitute Eqs. (11) and (12) into Eq. (10) we have

$$
\begin{aligned}
& \sum_{k=1}^{(n+1)(m+1)} \beta_{k}^{n m o} x_{k}^{n} y_{k}^{m}+\sum_{j=(n+1)(m+1)+1}^{K} \beta_{j}^{n m o} \sum_{k=1}^{(n+1)(m+1)} q_{j k}^{n m o} x_{k}^{n} y_{k}^{m} \\
& =\sum_{r=0}^{n} \sum_{s=0}^{m} \alpha_{r s}^{n m o} \sum_{k=1}^{(n+1)(m+1)} C_{r s k}^{n m o} x_{k}^{n} y_{k}^{m},
\end{aligned}
$$

or after rearranging

$$
\begin{aligned}
& \sum_{k=1}^{(n+1)(m+1)}\left(\beta_{k}^{n m o} x_{k}^{n} y_{k}^{m}+\sum_{j=(n+1)(m+1)+1}^{K} \beta_{j}^{n m o} q_{j k}^{n m o} x_{k}^{n} y_{k}^{m}\right) \\
& =\sum_{k=1}^{(n+1)(m+1)} \sum_{r=0}^{n} \sum_{s=0}^{m} \alpha_{r s}^{n m o} C_{r s k}^{n m o} x_{k}^{n} y_{k}^{m} .
\end{aligned}
$$

An easy solution would be

$$
\beta_{k}^{n m o}=\widehat{\beta}_{k}^{n m o}=\sum_{r=0}^{n} \sum_{s=0}^{m} \alpha_{r s}^{n m o} C_{r s k}^{n m o}, \quad k=1,2, \ldots(n+1)(m+1)
$$

and

$$
\beta_{k}^{n m o}=0, \quad k=(n+1)(m+1)+1, \ldots K
$$

However, such solution would, sometimes, result in large artificial higher-orders in the final kick map. So we follow Irwin's minimization method to introduce a set of Lagrange multipliers $\lambda_{j}, j=(n+1)(m+1)+1, \ldots K$ so as to introduce $(k-(n+1)(m+1))$ constraints to eliminate the extra independent bases $x_{j}^{n} y_{j}^{m}$, $j=(n+1)(m+1)+1, \ldots K$ for a unique solution of $\beta_{k}^{n m o}, k=1,2, \ldots K$. The Lagrange multipliers are introduced to minimize the sum of the squares of $\beta_{k}^{n m o}$, $k=1,2, \ldots K$. 
With the introducing of the Lagrange multiplier, an equally valid solution as opposing to Eq. (14) would be

$$
\begin{gathered}
\beta_{k}^{n m o}=\widehat{\beta}_{k}^{n m o}-\sum_{j=(n+1)(m+1)+1}^{K} \lambda_{j} q_{j k}^{n m o} \quad k=1,2, \ldots(n+1)(m+1) \\
\beta_{k}^{n m o}=\lambda_{k},
\end{gathered}
$$

that is, let the coefficients $\beta_{k}^{n m o}, k=(n+1)(m+1)+1, \ldots K$, of the extra independent rotation bases be Lagrange multipliers instead of 0 given in Eq. (14). Since these Lagrange multipliers are introduced to minimized the sum of the squares of $\beta_{k}^{n m o}$, [2] $k=1,2, \ldots K$. We therefore got $k-(n+1)(m+1)$ constraints by setting the derivative with respect to each of the $\lambda_{k}, k=(n+1)(m+1)+$ $1, \ldots K$, as follows:

$$
\begin{gathered}
\sum_{k=1}^{(n+1)(m+1)}\left(\widehat{\beta}_{k}^{n m o}-\sum_{l=(n+1)(m+1)+1}^{K} \lambda_{l} q_{l k}^{n m o}\right) q_{j k}^{n m o}+\lambda_{j}=0 \\
\quad \text { for } j=(n+1)(m+1)+1,(n+1)(m+1)+2, \ldots K
\end{gathered}
$$

Equation (16) has a total of $K-(n+1)(m+1)$ linear equations of $K-(n+1)(m+1)$ variables $\lambda_{j}$ 's. The linear system can be easily solved using standard numerical libraries. We therefore get a unique solution for $\beta_{k}^{n m o}$ given by Equation (15). Note that other combinations of $n, m, o$ such that $n+m+o=3$ can be obtained by following the above process. Once we have gotten all the $\beta_{k}^{n m o}$ 's for $k=1,2, \ldots K$, and all possible $n, m, o$, such that $n+m+o=3$, we have gotten ${ }_{2} g_{k}\left(\overrightarrow{\mathbf{x}}_{k}+\right)$ for $k=1,2, \ldots K$ such that

$$
\sum_{k=1}^{K}{ }_{2} g_{k}\left(\overrightarrow{\mathbf{x}}_{k}+\right)=f_{2}(\overrightarrow{\mathbf{z}}-)
$$

that is, the kick factorization for the 2 nd-order Lie factor is finished. 


\section{KICK FACTORIZATION OF THIRD-ORDER}

\section{DRAGT-FINN FACTOR}

Now that we have gotten

$$
\begin{aligned}
{ }_{2} m_{g}=\exp (: & \left.{ }_{2} g_{1}\left(\overrightarrow{\mathbf{x}}_{1}+\right):\right) \exp \left(:{ }_{2} g_{2}\left(\overrightarrow{\mathbf{x}}_{2}+\right):\right) \\
& \ldots \exp \left(:{ }_{2} g_{k}\left(\overrightarrow{\mathbf{x}}_{k}+\right):\right) \ldots \exp \left(:{ }_{2} g_{K}\left(\overrightarrow{\mathbf{x}}_{K}+\right):\right),
\end{aligned}
$$

our next step is to expand ${ }_{2} m_{g}$ into a Taylor map and then convert the Taylor map into a Dragt-Finn Factorization map up to 3rd order. Let it be

$$
{ }_{2} m_{g}=e^{\dot{2}_{2} f_{2}^{g}(\overrightarrow{\mathrm{z}}-):} e^{\dot{i}_{2} f_{3}^{g}(\overrightarrow{\mathrm{z}}-):}+\sigma(4)
$$

Then we have

$$
{ }_{2} f_{2}^{g}(\overrightarrow{\mathbf{z}}-)=f_{2}(\overrightarrow{\mathbf{z}}-)
$$

and

$$
{ }_{3} f_{3}(\overrightarrow{\mathbf{z}}-)=f_{3}(\overrightarrow{\mathbf{z}}-)-{ }_{2} f_{3}^{g}(\overrightarrow{\mathbf{z}}-) \neq 0 \text { in general. }
$$

The task in this section is to find ${ }^{3} g_{k}\left(\overrightarrow{\mathbf{x}}_{k}+\right), k=1,2, \ldots K$ such that

$$
\sum_{k=1}^{K}{ }^{3} g_{k}\left(\overrightarrow{\mathbf{x}}_{k}+\right)={ }_{3} f_{3}(\overrightarrow{\mathbf{z}}-)
$$

This can be done by following the similar process discussed in section 5 . Then add ${ }^{3} g_{k}\left(\overrightarrow{\mathbf{x}}_{k}+\right)$ to ${ }_{2} g_{k}\left(\overrightarrow{\mathbf{x}}_{k}+\right)$ for $k=1,2, \ldots K$ to get

$$
\begin{aligned}
{ }_{3} m_{g}=\exp \left(:{ }_{3} g_{1}\left(\overrightarrow{\mathbf{x}}_{1}+\right):\right) \exp \left(:{ }_{3} g_{2}\left(\overrightarrow{\mathbf{x}}_{2}+\right):\right) \\
\quad \ldots \exp \left(:{ }_{3} g_{k}\left(\overrightarrow{\mathbf{x}}_{k}+\right):\right) \ldots \exp \left(:{ }_{3} g_{K}\left(\overrightarrow{\mathbf{x}}_{K^{-}}+\right):\right)
\end{aligned}
$$

where

$$
{ }_{3} g_{k}\left(\overrightarrow{\mathbf{x}}_{k}+\right)={ }_{2} g_{k}\left(\overrightarrow{\mathbf{x}}_{k}+\right)+{ }^{3} g_{k}\left(\overrightarrow{\mathbf{x}}_{k}+\right), \quad k=1,2,3, \ldots K
$$




\section{KICK FACTORIZATION UP TO $\Omega$ ORDER}

Assume we have gotten

$$
\begin{array}{r}
{ }_{i} m_{g}=\exp \left(:_{i} g_{1}\left(\overrightarrow{\mathbf{x}}_{1}+\right):\right) \exp \left(:_{i} g_{2}\left(\overrightarrow{\mathbf{x}} F_{2}+\right):\right) \\
\quad \ldots \exp \left(:_{i} g_{k}\left(\overrightarrow{\mathbf{x}}_{k}+\right):\right) \ldots \exp \left(:{ }_{i} g_{K}\left(\overrightarrow{\mathbf{x}}_{K}+\right):\right)
\end{array}
$$

Following the similar process in section 6 , we would first expand ${ }_{i} m_{g}$ into a Taylor map and then extract from the Taylor map a Dragt-Finn Factorization map up to $i+1$ order, i.e. we would have

$$
\begin{aligned}
{ }_{i} m_{g}=\exp \left(:_{i} f_{2}^{g}(\overrightarrow{\mathbf{z}}-):\right) \exp \left(:_{i} f_{3}^{g}(\overrightarrow{\mathbf{z}}-):\right) \\
\quad \ldots \exp \left(:_{i} f_{i}^{g}(\overrightarrow{\mathbf{z}}-):\right) \exp \left(:_{i} f_{i+1}^{g}(\overrightarrow{\mathbf{z}}-):\right)+\sigma(i+2)
\end{aligned}
$$

where

$$
{ }_{i} f_{j}^{g}(\overrightarrow{\mathbf{z}}-)=f_{j}(\overrightarrow{\mathbf{z}}-) \quad \text { for } j=2,3, \ldots i
$$

and

$$
{ }_{i+1} f_{i+1}(\overrightarrow{\mathbf{z}}-)=f_{i+1}(\overrightarrow{\mathbf{z}}-)-{ }_{i} f_{i+1}^{g}(\overrightarrow{\mathbf{z}}-) \neq 0 \text { in general. }
$$

The task is then to follow the process in section 5 to find ${ }^{i+1} g_{k}\left(\vec{x}_{k}+\right)$, $k=1,2, \ldots K$, such that

$$
\sum_{k=1}^{K}{ }^{i+1} g_{k}\left(\overrightarrow{\mathbf{x}}_{k}+\right)={ }_{i+1} f_{i+1}(\overrightarrow{\mathbf{z}}-)
$$

Then we get

$$
\begin{aligned}
{ }_{i+1} m_{g}=\exp \left(:_{i+1} g_{1}\left(\overrightarrow{\mathbf{x}}_{1}+\right):\right) \exp \left(:_{i+1} g_{2}\left(\overrightarrow{\mathbf{x}}_{2}+\right):\right) \\
\quad \ldots \exp \left(:_{i+1} g_{k}\left(\overrightarrow{\mathbf{x}}_{k}+\right):\right) \ldots \exp \left(:_{i+1} g_{K}\left(\overrightarrow{\mathbf{x}}_{K}+\right):\right)
\end{aligned}
$$

where

$$
{ }_{i+1} g_{k}\left(\overrightarrow{\mathbf{x}}_{k}+\right)={ }_{i} g_{k}\left(\overrightarrow{\mathbf{x}}_{k}+\right)+{ }^{i+1} g_{k}\left(\overrightarrow{\mathbf{x}}_{k}+\right), \quad k=1,2, \ldots K
$$


Iterate the above process until $i+1=\Omega$. We therefore have the original closedorbit Taylor map be represented by a kick factorization map as follows:

$$
m=\mathcal{M}_{1}^{-1} \mathcal{M}_{1}^{\prime-1} \mathcal{M}_{R} m_{g} \mathcal{M}_{1}^{\prime} \mathcal{M}_{1}
$$

where

$$
m_{g}={ }_{\Omega} m_{g}=\exp \left(:{ }_{\Omega} g_{1}\left(\overrightarrow{\mathbf{x}}_{1}+\right):\right) \exp \left(:{ }_{\Omega} g_{2}\left(\overrightarrow{\mathbf{x}}_{2}+\right):\right) \ldots \exp \left(:{ }_{\Omega} g_{k}\left(\overrightarrow{\mathbf{x}}_{k}+\right):\right)
$$

or simply written as

$$
m_{g}=\exp \left(: g_{1}\left(\overrightarrow{\mathbf{x}}_{1}\right):\right) \exp \left(: g_{2}\left(\overrightarrow{\mathbf{x}}_{2}+\right):\right) \ldots \exp \left(: g_{k}\left(\overrightarrow{\mathbf{x}}_{k}+\right):\right)
$$

\section{KICK MAP TRACK}

Equation (21) can be further transformed by taking the rotation out of the Lie operators to get a global variable form, that is,

$$
m_{g}=\left(\mathcal{R}_{1} e^{: \hat{g}_{1}(x, y, \delta)}: \mathcal{R}_{1}^{-1}\right) \ldots\left(\mathcal{R}_{k} e^{: \hat{g}_{k}(x, y, \delta)}: \mathcal{R}_{k}^{-1}\right)\left(\mathcal{R}_{K} e^{: \hat{g}_{K}(x, y, \delta):} \mathcal{R}_{K}^{-1}\right)
$$

where $\mathcal{R}_{k}$ and $\mathcal{R}_{k}^{-1}, k=1,2, \ldots K$, are 4 -dimensional (phase space) rotations. We could combine adjacent rotations to get

$$
m_{g}=\mathcal{R}_{1} e^{: \hat{g}_{1}(x, y, \delta):} \mathcal{R}_{12} e^{: \hat{g}_{2}(x, y, \delta):} \ldots \mathcal{R}_{(k-1) k} e^{: \hat{g}_{k}(x, y, \delta):} \mathcal{R}_{k}^{-1} \ldots \mathcal{R}_{(K-1)}
$$

Since $\hat{g}_{k}$ is independent of conjugate momentums $\left(P_{x}, P_{y}, P_{\delta}\right)$, to advance the momentum we simply have

$$
\begin{aligned}
& \Delta P_{x}^{k}=\frac{\partial \hat{g}_{k}}{\partial x} \\
& \Delta P_{y}^{k}=\frac{\partial \hat{g}_{k}}{\partial y} \\
& \Delta P_{\delta}=\frac{\partial \hat{g}_{k}}{\partial \delta} \quad \text { for each } k=1,2, \ldots K
\end{aligned}
$$


Therefore,

$$
\begin{aligned}
& P_{x}=P_{x}+\Delta P_{x}^{k}(x, y, \delta) \\
& P_{y}=P_{y}+\Delta P_{y}^{k}(x, y, \delta) \\
& P_{\delta}=P_{\delta}+\Delta P_{\delta}^{k}(x, y, \delta) \quad \text { for each kick } k=1,2, \ldots K
\end{aligned}
$$

where $\Delta P_{x}, \Delta P_{y}, \Delta P_{\delta}$ are given as explicit functional form of $x, y, \delta$. Since at the beginning of each turn (or after each RF cavity), the off-momentum $\delta$ is updated and fixed before the next RF cavity, we can substitute the updated $\delta$ into each of the functional form of $\Delta P_{x}^{k}, \Delta P_{y}^{k}, \Delta P_{\delta}^{k}$ after each of the RF cavity to convert them into 2 -variable polynomial instead of 3 -variable polynomial to save computer time, that is, after substituting $\delta$ into the polynomial in the beginning of each term we have

$$
\begin{aligned}
& P_{x}=P_{x}+\Delta P_{x}^{k}(x, y) \\
& P_{y}=P_{y}+\Delta P_{y}^{k}(x, y) \\
& P_{\delta}=P_{\delta}+\Delta P_{\delta}^{k}(x, y)
\end{aligned}
$$

in each of the kicks to update the conjugate momenta of $x, y, \delta$. Therefore the nonlinear map $m_{g}$ becomes rotate, kick, rotate, kick, ..., rotate, kick, rotate for $k+1$ 4-Dimensional (phase space) rotation and $k 3$-dimensional kicks. The linear part are 6-Dimensional. All 6-Dimension coordinates are updated by 6-D matrix operation. 


\section{REFERENCES}

1. A. Dragt and J. Finn, J. Math. Phys., 20, 2649 (1979); A. Dragt et al., Ann. Rev. Nucl. Part. Sci., 38, 455 (1988).

2. J. Irwin, "A Multi-Kick Factorization Algorithm for Nonlinear Maps," SSC-228, 1989.

3. K. LaMon, LBL-XXX (1989).

4. Y. Yan, Zmap-A Differential-Algebra Map Extraction Program Using ZLIB, Superconducting Super Collider Publication No. SSCL-299 (1990).

5. Y. Yan and C. Yan, ZLIB-A Numerical Library for Differential Algebra and Lie Algebraic Treatment of Beam Dynamics, Superconducting Super Collider Publication No. SSCL-300 (1990).

\section{DISCLAIMER}

This report was prepared as an account of work sponsored by an agency of the United States Government. Neither the United States Government nor any agency thereof, nor any of their employees, makes any warranty, express or implied, or assumes any legal liability or responsibility for the accuracy, completeness, or usefulness of any information, apparatus, product, or process disclosed, or represents that its use would not infringe privately owned rights. Reference herein to any specific commercial product, process, or service by trade name, trademark, manufacturer, or otherwise does not necessarily constitute or imply its endorsement, recommendation, or favoring by the United States Government or any agency thereof. The views and opinions of authors expressed herein do not necessarily state or reflect those of the United States Government or any agency thereof. 